\title{
On the secondary star of the cataclysmic variable 1RXS J094432.1+035738
}

\author{
R. E. Mennickent ${ }^{1, \star}$, G. Tovmassian ${ }^{2}$, S. V. Zharikov ${ }^{2}$, C. Tappert ${ }^{1}$, J. Greiner $^{3}$, \\ B. T. Gänsicke ${ }^{4}$, and R. E. Fried ${ }^{5}$ \\ 1 Dpto. de Fisica, Fac. de Cs. Fisicas y Mat., Universidad de Concepcion, Casilla 160-C, Concepcion, Chile \\ 2 Observatorio Astronomico Nacional, Instituto de Astronomia, UNAM, 22860 Ensenada, Mexico \\ 3 Astrophysical Institute Potsdam, An der Sternwarte 16, 14482 Potsdam, Germany \\ 4 Universitats-Sternwarte, Geismarlandstr. 11, 37083 Gottingen, Germany \\ 5 Braeside Observatory, PO Box 906, Flagstaff, AZ 86002, USA
}

Received 18 October 2001 / Accepted 18 December 2001

\begin{abstract}
We present $V$ and $R_{\mathrm{c}}$-band photometry and optical near-infrared spectroscopy of the cataclysmic variable 1RXS J094432.1+035738. We detected features of a cool secondary star, which can be modeled with a red dwarf of spectral type $\mathrm{M} 2_{-1.0}^{+0.5} \mathrm{~V}$ at a distance of $433 \pm 100 \mathrm{pc}$.
\end{abstract}

Key words. stars: individual: 1RXS J094432.1+035738 - novae - cataclysmic variables stars: fundamental parameters - stars: evolution - binaries: general

\section{Introduction}

The optical counterpart of the ROSAT source 1RXS J094432.1+035738 was identified as a cataclysmic variable $(\mathrm{CV})$ in the course of follow-up observations of $\mathrm{CV}$ candidates from the Hamburg Quasar Survey (Jiang et al. 2000). These authors presented a spectrum showing Balmer and HeI emission, typical of a cataclysmic variable star (Warner 1995). Two outbursts have been observed and recorded by VSNET observers in January 2001 and June 2001 (http://www.kusastro.kyoto-u.ac.jp/vsnet/), suggesting a dwarf nova nature for the variable. While writing this paper, we were informed that Thorstensen $\&$ Fenton found an orbital period of $0.1492 \pm 0.0004 \mathrm{~d}$ for this object (vsnet-alert 5988, Thorstensen, private communication). Here we present a detailed analysis of the spectrum of this target, including the first detection of the secondary star, the analysis of the radial velocity of the $\mathrm{H} \alpha$ emission line and a refinement of the orbital period.

Send offprint requests to: R. E. Mennickent, e-mail: rmennick@stars.cfm.udec.cl

* Based on observations obtained at the European Southern Observatory, ESO proposal 65.H-0410(A).

\section{Observations and data reduction}

\subsection{Optical photometry}

We obtained differential photometry of 1RXSJ094432.1+ 035738 at Braeside Observatory, Arizona, using a $41 \mathrm{~cm}$ reflector equipped with a SITe 512 CCD camera, during the nights of March 11 and 12, 2000 (UT). We also obtained $R_{\mathrm{c}}$-band time-resolved photometry during two nights in April 17-18, 2001 (UT) at the $1.5 \mathrm{~m}$ telescope of the Observatorio Astronomico Nacional de San Pedro Martir (OAN SPM), Baja California, Mexico. This telescope was equipped with a $1024 \times 1024$ pixel SITe CCD. The images were corrected for bias and flat field. For the observations of April, 2001, aperture differential photometry was carried out using the comparison star located at $\alpha_{2000}=$ 09:44:27.25 and $\delta_{2000}=03: 58: 09.9$ and the check star located at $\alpha_{2000}=09: 44: 26.45$ and $\delta_{2000}=03: 57: 42.7$. An estimate of the uncertainty of the CCD photometry was obtained from the standard deviation of the differential light curve between comparison and check star, viz. 0.02 mag. Since comparison and check stars are slightly fainter than the variable, the photometric error in this case is dominated by the fainter star (i.e. the check star) and the above figure is an upper limit for the uncertainty of the variable minus comparison light curve (e.g. Howell et al. 1988). We did not obtain an absolute calibration for our photometric dataset obtained at San Pedro Martir. However, for the 
Table 1. Journal of spectroscopic observations. Telescope and grating, resolution and number of spectra are given. Zero point for Heliocentric Julian Day is $\mathrm{HJD}_{0}=2451600$.

\begin{tabular}{ccccc}
\hline Tel. & Grating & Res. $(\AA)$ & HJD & $\mathrm{N}$ \\
\hline $2.12 \mathrm{~m} / \mathrm{SPM}$ & $300 \mathrm{l} / \mathrm{mm}$ & 8.0 & $45.6676-7395$ & 11 \\
$1.54 \mathrm{~m} / \mathrm{ESO}$ & $\# 5$ & 7.5 & $94.5003-5759$ & 6 \\
$1.54 \mathrm{~m} / \mathrm{ESO}$ & $\# 7$ & 4.0 & $95.4913-5675$ & 17 \\
\hline
\end{tabular}

observations carried out at Braeside Observatory, we obtained $V$ magnitudes of 1RXS J094432.1+035738 relative to the $V=11.82$ comparison star GSC0023900958.

\subsection{Spectrophotometric observations}

The spectrophotometric observations were conducted at the $2.12 \mathrm{~m}$ telescope of the Observatorio Astronomico Nacional, San Pedro Martir, Mexico, during April 12, 2000 (UT), and at the 1.54 Danish Telescope of La Silla European Southern Observatory, on May 29/30, 2000 (UT). Standard IRAF tasks were used for flat field correction, bias subtraction, cosmic ray removal, extraction and wavelength-flux calibration.

We used the Boller \& Chivens spectrograph installed in the Cassegrain focus of the $2.12 \mathrm{~m}$ telescope at OAN SPM. The 300 lines/mm grating was used to cover a wavelength range from 3700 to $7600 \AA$. The TEK $1024 \times 1024$ pixel CCD with a $0.24 \mu \mathrm{m}$ pixel size was attached to the spectrograph.

The Danish Faint Object Spectrograph and Camera (DFOSC) was used with grisms 5 and 7 at the Danish telescope in Chile, yielding a combined wavelength range of 3500-9500 $\AA$. A slit width of 1.5 arcsec was chosen in order to match the typical point spread function at the focal plane of the telescope. This resulted in spectral resolutions of $4 \AA$ (grism 7) and $7.5 \AA$ (grism 5). The journal of observations is given in Table 1.

In general, the wavelength calibration functions were constructed with $\approx 40 \mathrm{He}-\mathrm{Ne}$ lines and had typical rms of $0.1 \AA\left(5 \mathrm{~km} \mathrm{~s}^{-1}\right.$ at $\left.\mathrm{H} \alpha\right)$.

Spectrophotometric standard stars (Feige 34 and HZ 44 in OAN SPM and LTT 3864 and LTT 7987 in La Silla, Hamuy et al. 1992; Hamuy et al. 1994) were observed in order to perform flux calibration. To minimize slit losses and improve flux calibration, a wide slit was used for standards stars $\left(5^{\prime \prime}\right)$ while the slit for all objects was aligned along the paralactic angle. The atmospheric absorption bands were removed from the spectra using the "telluric" IRAF task. For that we used a template obtained by normalizing the standard star spectrum to the continuum, and interpolating the resulting spectrum between the hydrogen absorption lines intrinsic to the early spectral type.

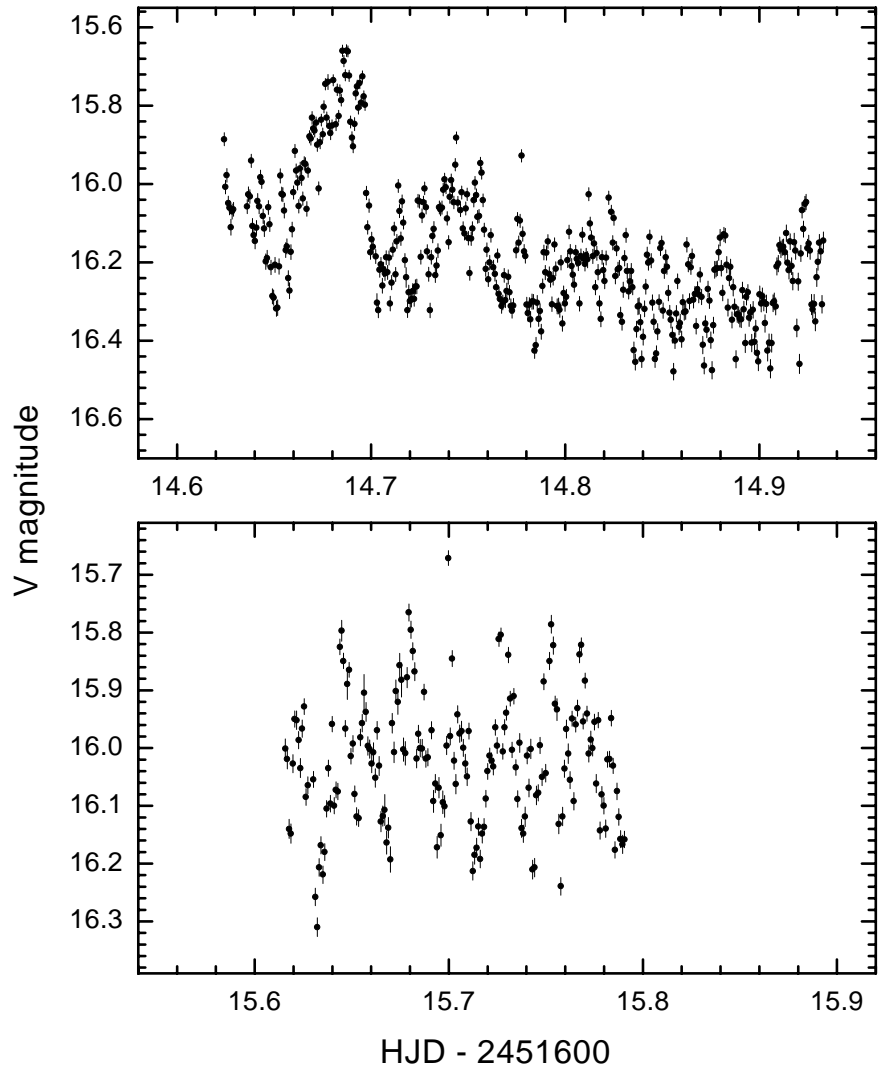

Fig. 1. $V$ magnitudes and their errors for the observations at Braeside Observatory.

\section{Results}

\subsection{Photometry}

Figures 1 and 2 show the light curves of 1RXS J094432.1+035738. From these figures, the remarkable variability of this star is evident. The bottom panel of Fig. 1 reveals non-coherent variability with amplitude $0.4 \mathrm{mag}$ and peak-to-peak time scales of 1 hour, whereas in the upper panel a complex behaviour is seen; a long-term oscillation with a time scale of 1.7 hours over a long-term fading and flickering in time scales longer than 10 minutes. Regarding the data taken in San Pedro Martir Observatory (Fig. 2), discarding the data previous to the maximum at 2452016.6905 , and shifting the remaining data to a common nightly mean, we find a good model with a sinusoid with a period of $0.111 \pm$ 0.005 days $(2.66 \pm 0.12 \mathrm{~h})$. A frequency analysis of the whole dataset did not yield any conclusive periodicity.

\subsection{Spectrum description and radial velocities}

Our averaged spectrum shown in Fig. 3 reveals a rather flat continuum reaching a maximum around $7500 \AA$ and decreasing to longer wavelengths. Balmer, Paschen (not shown in Fig. 3 since the flux calibration at these wavelengths is not reliable) and HeI emission lines are also present. At our resolution, these lines are single peaked and the Balmer decrement rather flat. From the 


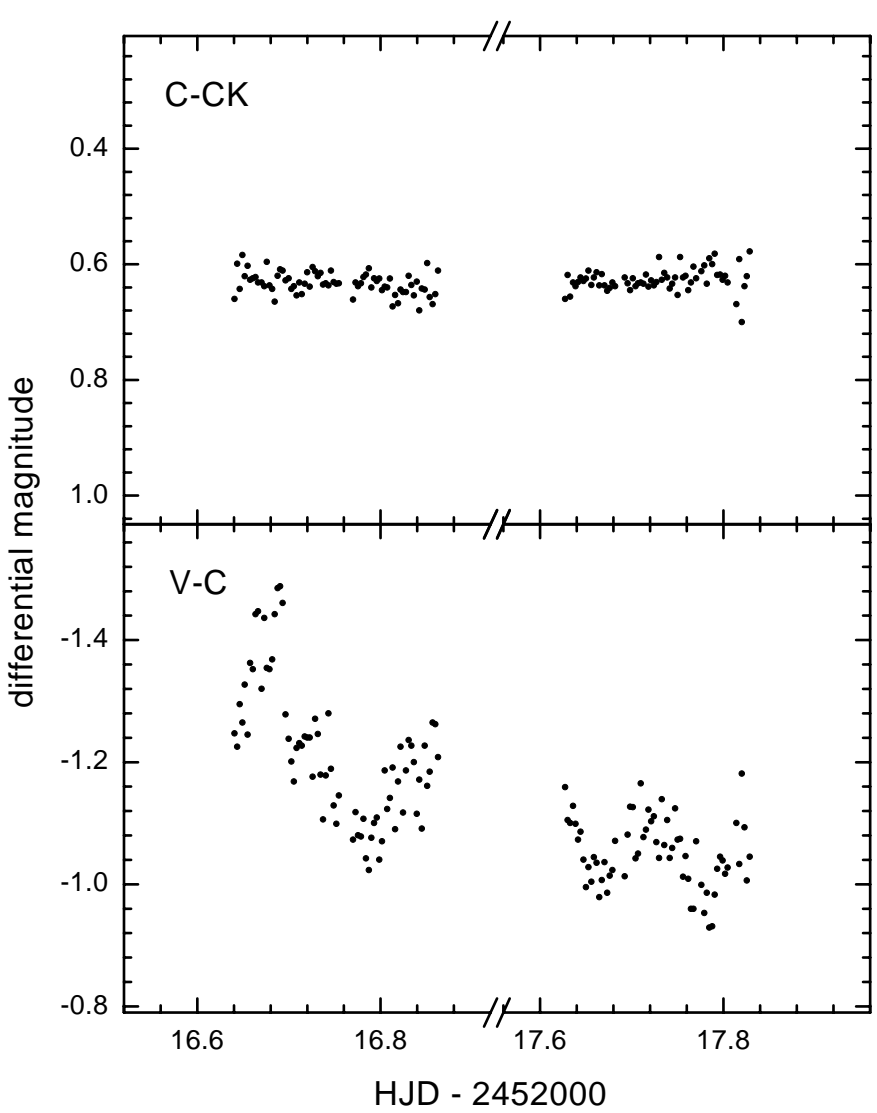

Fig. 2. Differential magnitudes of comparison and check star (upper panel) and variable and comparison star (bottom panel) for the San Pedro Martir Observatory observations.

spectrum we derived spectrophotometric magnitudes of $V=16.7 \pm 0.1$ for April 2000 and $V=16.4 \pm 0.1$ for May 2000 , including correction factors by slit looses derived for the finite slit width. The above figures indicate that the object was observed in quiescence in both observing sessions. Contrasting with the spectrum shown by Jiang et al. (2000), we observe the TiO absorption bands typical of M type stars.

We give in Table 2 the fluxes and equivalent widths for the main emission lines detected in the averaged spectrum. Typical errors are of the order of $10 \%$. The average of the full width at half intensity for hydrogen lines is $1580 \pm 235 \mathrm{~km} \mathrm{~s}^{-1}$ and for neutral helium lines is $1340 \pm 270 \mathrm{~km} \mathrm{~s}^{-1}$. The mean $\mathrm{H} \alpha$ equivalent width was $-59 \pm 3 \AA$ on April 2000 , and $-50 \pm 4 \AA$ on May 2000. The corresponding figures for HeI 5875 were $-15 \pm 1 \AA$ and $-14 \pm 2 \AA$, respectively. We compared the observed Balmer decrements with theoretical results by Williams (1991), who modeled optically thin gas in the emission lines in accretion disks. He computed $\mathrm{H} \beta$ strengths and Balmer decrements for a grid of temperatures, inclinations and mid-plane accretion disk density $\left(N_{0}\right.$ in units of nucleons per $\mathrm{cm}^{3}$ ). We find that the observed $D(\mathrm{H} \alpha / \mathrm{H} \beta)=1.35$ and $D(\mathrm{H} \gamma / \mathrm{H} \beta)=0.86$ nicely fit the results for the $T=15000 \mathrm{~K}, \log N_{0}=12.5$ model.

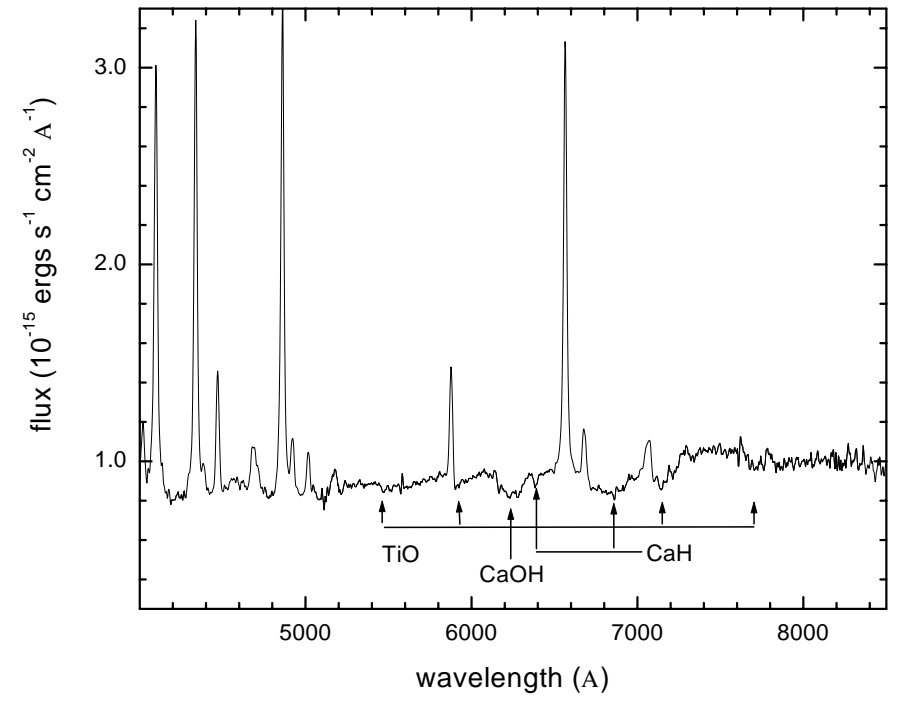

Fig. 3. Combined spectrum of 1RXS J094432.1+035738. Identified absorption bands are labeled.

Table 2. Spectral lines detected in the spectrum of 1RXS J094432.1+035738. We give the equivalent width in $\AA$ and the flux in $10^{-15} \mathrm{erg} \mathrm{s}^{-1} \mathrm{~cm}^{-2} \AA^{-1}$.

\begin{tabular}{lrc}
\hline \multicolumn{1}{c}{ Line } & EW & flux \\
\hline H10 & N/A & 2.1 \\
H9/He I 3828 & -9 & 2.2 \\
H8 & -20 & 2.4 \\
H $\epsilon$ 3963 & -37 & 2.7 \\
He I 4023 & -5 & 1.1 \\
H $\delta$ & -65 & 2.8 \\
H $\gamma$ & -70 & 3.0 \\
He I 4388 & -4 & 0.9 \\
He I 4471 & -15 & 1.4 \\
He II 4685 / He I 4713 & -11 & 1.0 \\
H $\beta$ & -72 & 3.1 \\
He I 4920 & -8 & 1.0 \\
He I 5015 & -4 & 1.0 \\
He I 5875 & -14 & 1.5 \\
H $\alpha$ & -65 & 3.1 \\
He I 6678 & -8 & 1.2 \\
He I 7065 & -8 & 1.1 \\
H I 8863 & -5 & N/A \\
H I 9014 & -9 & N/A \\
H I 9229 & -13 & N/A \\
\hline
\end{tabular}

We measured the radial velocities of the $\mathrm{H} \alpha$ emission line in the single spectra by fitting the profile with a Gauss function. We observe a radial velocity maximum at HJD 2451645.6872 (April 12, 2000) followed by a smooth velocity decrease. On the other hand, on May 23, 2000, we observe a velocity minimum at HJD 2451695.4992 followed by a maximum at HJD 2451695.5586 . A pdm periodogram for our radial velocity dataset indicates several possible solutions. Our velocities are not distributed in time in such way as to decide about these possible aliases. However, the data provided by Thorstensen \& Fenton (private comunication) is well distributed in time to 


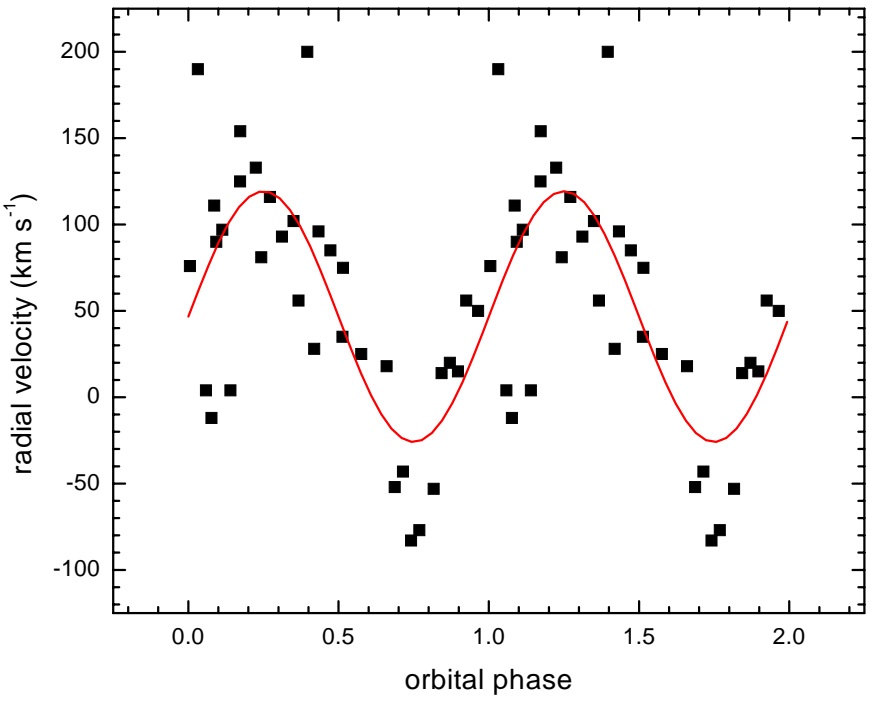

Fig. 4. The $\mathrm{H} \alpha$ emission line radial velocities folded with the $0.14892 \mathrm{~d}$ period. The best sinus fit, with half-amplitude $73 \pm$ $8 \mathrm{~km} \mathrm{~s}^{-1}$ and zero point $47 \pm 6 \mathrm{~km} \mathrm{~s}^{-1}$, is also shown. The time for radial velocity croosing from blue to red coresponds to $\mathrm{HJD}=2451645.6538(30)$.

determine the daily cycle count. We therefore assumed the Thorstensen \& Fenton period (0.1492 $\pm 0.0004 \mathrm{~d})$ and tried to refine it by doing a pdm analysis inside the period error window provided by these authors. The result indicates two solutions with similar statistical significance, viz. $0.14892 \pm 0.00013 \mathrm{~d}$ and $0.14936 \pm 0.00013 \mathrm{~d}$. The period error above corresponds to the half width at half maximum of the main peak in the power window. In both cases, the $\mathrm{H} \alpha$ radial velocity curve can be described by a sine law with half-amplitude around $75 \mathrm{kms}^{-1}$ (Fig. 5). The fact that $K_{1}$ is moderately high, and the light curve does shows variations, but no eclipses, point to a medium inclination in the range of 30-60 degrees.

\subsection{Modeling the spectral energy distribution: The secondary star revealed}

The existence of the $\mathrm{TiO}$ band at $5450 \AA$ sets a low limit for the spectral type of the secondary star of M 0.5, whereas the absence of the $\mathrm{TiO}$ band at $8400 \AA$ sets an upper limit of M 5 (e.g. Reid \& Hawley 2000). In order to better constrain the spectral type of the secondary star, we fitted the continuum with a composite spectrum consisting of the contributions of a power-law continuum and a late type template spectrum:

$$
S(\lambda)=a \times T(\lambda)+b \times \lambda^{c}
$$

where $S$ is the observed spectrum, $T$ the red dwarf template spectrum, $\lambda$ the wavelength in Angströms and $a, b, c$ parameters to be found. Of course, $a$ and $b$ are constrained to the positive domain. The data sampling for fitting was selected avoiding emission lines. A sequence of template spectral types between M0V and M4V were

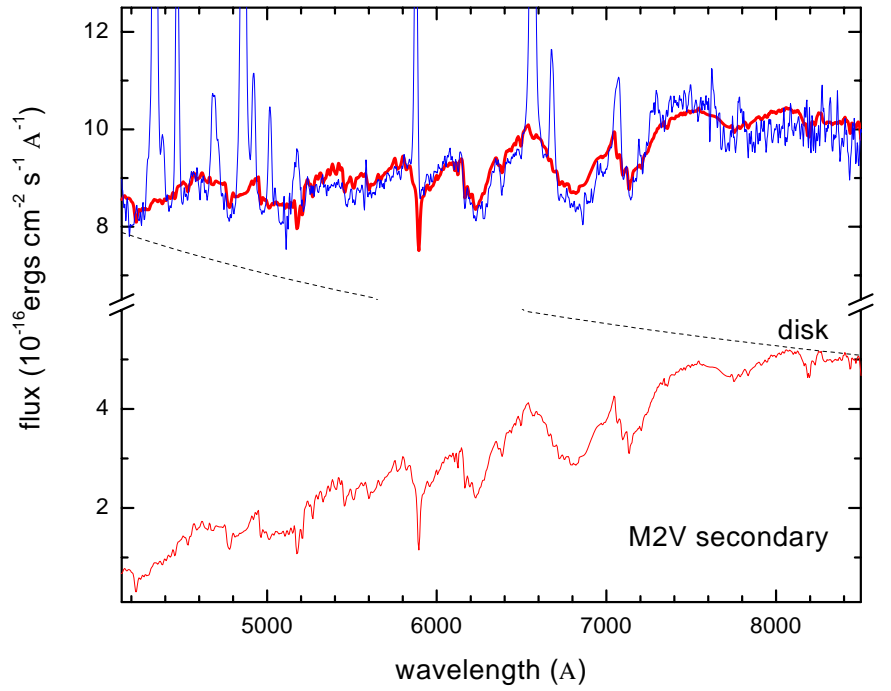

Fig. 5. The observed spectrum, the best disk and secondary star model and the sum of them (thick line). Spectral regions with emission lines were excluded in the fitting process.

obtained from Pickles (1998), which are available online at the CDS (Centre de Données astronomiques de Strasbourg) database (http://cdsweb.u-strasbg.fr). We run a Levenberg-Marquardt non-linear least squares fitter getting the $a, b, c$ parameters and the $\chi^{2}$ for every template. Our results indicated that the best fit is reached with a template of spectral type $\mathrm{M} 2 \mathrm{~V}$ and a power law with slope $c=-0.61(2)$. The template with a spectral type of M0V gives $\chi^{2}$ larger by a factor 2.2 , whereas the templates with spectral types M1V, M3V and $\mathrm{M} 4 \mathrm{~V}$ give $\chi^{2}$ larger than the $\mathrm{M} 2 \mathrm{~V}$ template by $5 \%$, $25 \%$ and $30 \%$, respectively. For the M2V model, shown in Fig. 5, the disk contributes $64 \%$ and the secondary star $36 \%$ to the total light at $\lambda 6700 \AA$.

\subsection{Distance estimates}

From the inferred Johnson- $V$ magnitude of the secondary star, viz. 18.04, we estimate a distance of $425{ }_{-47}^{+63} \mathrm{pc}$, assuming $M_{V}=9.9$ for the $\mathrm{M} 2 \mathrm{~V}$ secondary and an uncertainty in the spectral class of ${ }_{-1.0}^{+0.5}$ subtypes. An independent estimate can be obtained using the $M_{V}$ (outburst peak) vs. $P_{\mathrm{o}}$ relationship calibrated by Warner (1995, his Eq. (3.4)). Assuming a magnitude at maximum of $V=13.1$, based on the $V S N E T$ reports, we obtain a distance of $d=454$ pc. Finally, using the Beuermann \& Weichhold (1999) method to derive CV distances, which is based on the flux deficiency $f_{\mathrm{TiO}}$ at wavelengths 7500 and $7165 \AA$, i.e. independent of any disk contamination, we find distances of $399 \pm 96 \mathrm{pc}, 431 \pm 104 \mathrm{pc}$, and $427 \pm 103$ pc for $\mathrm{M} 1 \mathrm{~V}, \mathrm{M} 2 \mathrm{~V}$ and $\mathrm{M} 2.5 \mathrm{~V}$ type secondaries, yielding a mean of 419 pc. In the above calculation we assumed a radius for the secondary star of $0.36 \pm 0.09$ solar radii, accordingly to the orbital period of $3.58 \mathrm{~h}$ (Smith \& Dhillon 1998, Eq. (11)). The average of the three above estimates is $d=433 \mathrm{pc}$ with a likely uncertainty of $100 \mathrm{pc}$. 


\subsection{X-ray data}

Covered in the ROSAT survey for $243 \mathrm{~s}$ with a mean count-rate of 0.089 counts/s, the hardness ratio suggests a hard spectrum, possibly absorbed. Using a thermal bremsstrahlung model with a fixed temperature of $20 \mathrm{keV}$, we derive an unabsorbed X-ray luminosity of $8.8 \times 10^{29}$ $(d / 100 \mathrm{pc})^{2} \mathrm{erg} \mathrm{s}^{-1}$ in the $0.1-2.4 \mathrm{keV}$ ROSAT range. Using our distance estimate above, this figure corresponds to $1.65 \times 10^{31} \mathrm{erg} \mathrm{s}^{-1}$, a typical value for dwarf nova in quiescence (Cordova \& Mason 1984).

\section{Discussion: On the nature of 1RXS J094432.1+035738}

The orbital period found by Thorstensen \& Fenton (private comunication) is quite short for a dwarf nova above the period gap (Shafter 1992). However, the low He II $4686 / \mathrm{H} \beta$ emission line ratio, along with the absence of cyclotron harmonics in the spectrum, probably rule-out the hypothesis of a AM Her type system, which is also consistent with the available X-ray data. On the other hand, the presence of outbursts and the general spectrum appearance, suggests a dwarf nova type object. The short-term photometric variability observed in Fig. 1 is reminiscent of rotational modulations seen in intermediate polars, but the changing nature of this feature, and their non-coherent nature, makes this interpretation doubtful. The outburst amplitude, $\sim 3.5 \mathrm{mag}$, implies a recurrence time of around 30 days, accordingly to the Kukarkin-Parenago relationship (e.g. Warner 1995, Eq. (3.1)). The above figure suggests that many outbursts might have been missed by observers in previous campaigns (the two reported outburst are separated by 6 months). We argue that 1RXS J094432.1+035738 is very likely a U Geminorum type dwarf nova, although an intermediate polar nature cannot be discounted.

Acknowledgements. We acknowledge the referee, Dr. Klaus Beuermann, for useful comments that helped to improve a first version of this paper. We also acknowledge Dr. John Thorstensen for his cooperation in discussing the orbital period of this object. This work was supported by Grant Fondecyt 1000324 and DI UdeC 99.11.28-1.

\section{References}

Beuermann, K., \& Weichhold, M. 1999, in ASP Conf. Ser., 157, Annapolis Workshop on Magnetic Cataclysmic Variables, ed. C. Hellier, \& K. Mukai (San Francisco: ASP), 283

Cordova, F. A., \& Mason, K. O. 1984, MNRAS, 206, 879

Downes, R. A., Webbink, R. F., Shara, M. M., et al. 2001, PASP, 113, 764

Hamuy, M., et al. 1992, PASP, 104, 533

Hamuy, M., et al. 1994, PASP, 106, 566

Howell, S. B., Warnock, A. I., \& Mitchell, K. J. 1988, AJ, 95, 247

Jiang, X. J., Engels, D., Wei, J. Y., Tesch, F., \& Hu, J. Y. 2000, A\&A, 362, 263

Pickles, A. J. 1998, PASP, 110, 863

Reid, N., \& Hawley, S. 2000, New light on dark stars (SpringerPraxis Books in Astrophysics and Astronomy)

Shafter, A. W. 1992, ApJ, 394, 268

Thorstensen, J. R., \& Fenton, W. H. 2001, in ASP Conf. Ser., The Physics of Cataclysmic Variable Stars, in press

Vennes, S., Ferrario, L., \& Wickramasinghe, D. 1999, in ASP Conf. Ser. 157, Annapolis Workshop on Magnetic Cataclysmic Variables, ed. C. Hellier, \& K. Mukai (San Francisco: ASP), 143

Warner, B. 1995, Cataclysmic Variable Stars (Cambridge University Press)

Williams, G. 1991, AJ, 101, 1929 